\title{
Synthesis of 1,1-Dioxobenzo[b]thiophene-2-ylmethyloxycarbonyl (Bsmoc) Protected $N$-Methyl Amino Acids by Reduction of Bsmoc-5-Oxazoli- dinones and Their Use in Peptide Synthesis
}

\author{
Chennakrishnareddy Gundala, Subramanyam J. Tantry, Shankar A. Naik and \\ Vommina Venkata Sureshbabu*
}

Peptide Research Laboratory, Department of Studies in Chemistry, Central College Campus, Bangalore University, Dr.
B.R. Ambedkar Veedhi, Bangalore - 560001 , India

\begin{abstract}
The synthesis of Bsmoc- $N$-methyl amino acids is presented. The first step involves $p$-toluenesulfonic acid $(\mathrm{TsOH})$ catalysed condensation of a Bsmoc-amino acid with paraformaldehyde to furnish $N$-Bsmoc-5-oxazolidinone under MW irradiation. This intermediate is reduced to the corresponding $N$-methyl amino acid using triethylsilane $\left(\mathrm{Et}_{3} \mathrm{SiH}\right)$ and trifluoroacetic acid (TFA) at r.t. The $N$-methyl amino acids are converted into corresponding acid fluorides using diethylaminosulfur trifluoride (DAST) and employed as coupling agents in the synthesis of dipeptides. The peptide coupling was mediated by KOAt in $\mathrm{CH}_{2} \mathrm{Cl}_{2}$.
\end{abstract}

Keywords: Bsmoc $N$ methyl amino acids, 5-Oxazolidinone, Amino acid fluorides, KOAt.

\section{INTRODUCTION}

The $N$-methyl amino acids are wide spread in nature as part of larger peptidic natural products [1-3], alamethicin peptides and cyclosporine family [4]. They find broad application in the design and development of biologically potent molecules in medicinal chemistry $[5,6]$. In addition, they are inserted into biologically active peptides to enhance potency and duration of action or to convert an agonist to an antagonist or to obtain information about backbone conformation or to increase the aqueous solubility of a peptide [7]. For the purpose of peptide synthesis, it is essential to have $N$ urethane protected $N$-methyl amino acids as building blocks. Several procedures are available for the synthesis of $N$ carbamate protected $N$-methyl amino acids [8]. Benoiton procedure [9] involving $\mathrm{NaH}$ and $\mathrm{MeI}$ for abstraction of proton followed by methylation is the method of choice being utilized to prepare base stable Boc and Z protected $N$-methyl amino acids. An improvement has been made to the above method by using KH/18-crown ether and alkyl halide. BenIshai [10] demonstrated the first synthesis and utility of 5oxazolidinones as a tool for simultaneous protection of the amino and carboxyl groups. Recently, Sureshbabu et al. [11] demonstrated the utility of MW irradiation for the rapid, high yielding synthesis of 5-oxazolidinones. The entire protocol is complete in $2 \mathrm{~min}$. Govender and Arvidsson [12] further fine tuned by selecting acetonitrile in place of toluene as a solvent due to its poor dielectric heating capacity. Weinreb et al. [13] had developed a procedure for the reduction of methylol derivatives of simple amides using triethylsilane -

*Address correspondence to this author at the Peptide Research Laboratory, Department of Studies in Chemistry, Central College Campus, Bangalore University, Dr. B.R. Ambedkar Veedhi, Bangalore - 560 001, India; E-mail: hariccb@rediffmail.com trifluoroacetic acid. This involves reduction of $\mathrm{N}$-acyliminium ion followed by ionic hydrogenation. Freidinger et al. [14] is the first one to synthesize Fmoc- $N$-methylated amino acids via 5-oxazolidinones and their reduction employing $\mathrm{Et}_{3} \mathrm{SiH}-\mathrm{TFA}$. This procedure was extended for the synthesis of $N$-methyl Trp, Asn, His and Arg and then to all other amino acids by Aurelio et al. [15,16]. Zhang et al. [17] accomplished the reductive ring opening of the 5oxazolidinone's using Lewis acid catalysis $\left(\mathrm{AlCl}_{3}\right.$ or $\left.\mathrm{ZnBr}_{2}\right)$. This paper deals with the hitherto unreported Bsmoc- $N$ methyl amino acids synthesis employing Freidinger procedure.

Bsmoc group [18], introduced by Louis Carpino, for amino protection has significant advantages over the Fmoc group. The key difference of this invention is the mode of cleavage mechanism through Michael-like attack of base on $\alpha, \beta$ unsaturated sulfones [19] followed by elimination of $\mathrm{CO}_{2}$ and amino component (Scheme 1). As Bsmoc group can be effectively cleaved with very low concentration of a weak base, its usage can circumvent several base catalyzed side reactions. Upon cleavage of the Fmoc group, the acidic phosphate buffer wash is necessary for the removal of DBF adduct formed. On the other hand, the use of either water or brine wash is sufficient for the complete removal of all by products formed in Bsmoc chemistry. This leads to a clear phase separation and minimal loss of carefully built peptide chain into aqueous phase. Ultimately, increase in yields of the target peptides can be achieved. The utility of Bsmoc group in rapid, continuous solution-phase peptide synthesis was demonstrated by the synthesis of a human parathyroid hormone fragment (hPTH, 21-29) [20]. The synthesis of peptides employing acid fluorides is well known in peptide chemistry. In contrast to the corresponding acid chlorides, the acid fluorides have greater stability and are suitable for carrying out reactions bearing acid labile groups such as ${ }^{t} \mathrm{Bu}$, 
Boc or trityl groups in the side chain [21]. Acid fluorides have been shown to accomplish difficult esterifications such as between a weakly nucleophilic secondary alcohol and a cyclohexyl amino acid, where other methods of activation such as DCC/DMAP, mixed anhydride, BOP-Cl or BOP have failed [22]. Successful synthesis of acyl carrier protein 65-74 fragment (ACP), magainin-II-amide, human corticotropin-releasing factor $(h-\mathrm{CRF})$ and peptide related to $\mathrm{Alz}$ heimers disease $\mathrm{A} \beta$ (1-42) which are known as difficult sequences to synthesize, have been accomplished efficiently employing acid fluorides [23]. Very difficult sequence containing sterically hindered $C^{\alpha \alpha}$-dialkylamino acids such as Aib [24], 1-aminocyclohexane-1-carboxylic acid and 4amino-piperidine-4-carboxylic acid [25] have also been achieved with greater efficiency by the use of corresponding acid fluorides. A comparative study of activation methods for the synthesis of the model tetrapeptide H-Aib-Aib-AibAib-OH by symmetric anhydride, PyBroP, acid fluoride and UNCA methods revealed that the acid fluoride coupling perform exceptionally well [26]. The acid fluorides are synthesized using cyanuric fluoride in presence of pyridine according to the general technique of Olah [27]. An alternative procedure for the synthesis of Fmoc-amino acid fluorides involves DAST [28] which is advantageous owing to the formation of water soluble byproducts, thus facilitating the isolation of the acid fluorides. The synthesis of Bsmoc Nmethyl aminoacid fluorides using DAST and their coupling in the presence of KOAt is described.

\section{RESULTS AND DISCUSSION}

Towards the first step, benzothiophene-2-methanol, prepared using literature procedure, was oxidized using $m$ chloroperbenzoic acid ( $m$-CPBA) at r.t. A simple work up of the reaction resulted the sulfone in $88 \%$ yield. Earlier description of the oxidation involved in the use of excess of acetic acid and sodium perborate. During the preliminary rounds of preparation of sulfone we followed the reported protocol. In such crops, during the removal of acetic acid considerable amount of the product was decomposed resulting in low yield of sulfone. Treatment of 1,1-dioxo- benzothiophene-2-methanol with triphosgene resulted in $77 \%$ of Bsmoc- $\mathrm{Cl}$ (Scheme 2) which was then converted into its succinimide. Bsmoc- amino acids were prepared by the reported procedure .

For the synthesis of Bsmoc-amino acids, Bolin's technique of $\mathrm{N}, \mathrm{O}$-bis-trimethyl amino acids was employed. Bsmoc-OSu was added to a solution of freshly prepared $N, O$-bis-trimethyl amino acid in DCM and the reaction mixture was stirred at r.t. for $2 \mathrm{hr}$. Workup of the reaction mixture resulted in Bsmoc amino acids in good yield (Scheme 3).

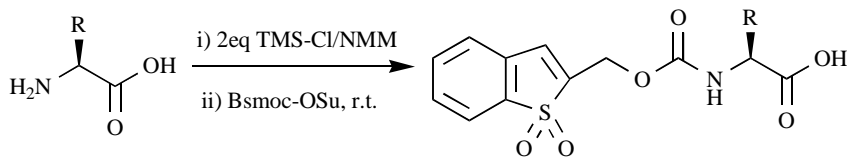

$\mathrm{R}=\mathrm{H}, \mathrm{CH}_{3}, \mathrm{CH}\left(\mathrm{CH}_{3}\right)_{2}, \mathrm{CH}_{2} \mathrm{C}_{6} \mathrm{H}_{5}$,

$\mathrm{CH}_{2} \mathrm{CH}\left(\mathrm{CH}_{3}\right)_{2}, \mathrm{CH}\left(\mathrm{C}_{2} \mathrm{H}_{5}\right) \mathrm{CH}_{3}$

Scheme 3. Synthesis of Bsmoc-amino acid.

The synthesis of Bsmoc-5-oxazolidinones was carried out starting from Bsmoc-amino acids. Bsmoc-amino acid was condensed with paraformaldehyde in presence of catalytic amount of $\mathrm{TsOH}$ under microwave irradiation. The reaction was complete within 3 min and workup of the reaction mixture resulted in 1a-f (Scheme 4, Table 1) in 89 to $95 \%$ yield. These oxazolidinones were stable at room temperature and had a strong IR stretching frequency at $1800 \mathrm{~cm}^{-1}$. Further, the ${ }^{1} \mathrm{H}$ NMR specta of the same also showed a signal in the vicinity of gamma amino alcohol $\delta 5.1 \mathrm{ppm}$ which is the characteristic peak of 5-oxazolidinone.

The 5-oxazolidinones were reduced into their corresponding $N$-methyl amino acids by treatment with 2.5 eq of $\mathrm{Et}_{3} \mathrm{SiH}$ in presence of TFA-CHCl $\mathrm{CH}_{3}(1: 1)$. This has resulted in simultaneous ring opening along with reduction to obtain Bsmoc- $N \mathrm{Me}$-amino acids $2 \mathrm{a}-\mathrm{f}$ in 80 to $93 \%$ yield (Scheme 5, Table 2). The reaction was complete after stirring for $22 \mathrm{hr}$ at r.t. The IR specta of Bsmoc- $N$-methyl amino acids showed the absence of IR stretching band at $1800 \mathrm{~cm}^{-1}$ corresponding to 5-oxazolidinones. The ${ }^{1} \mathrm{H}$ NMR of Bsmoc- $N$-methyl

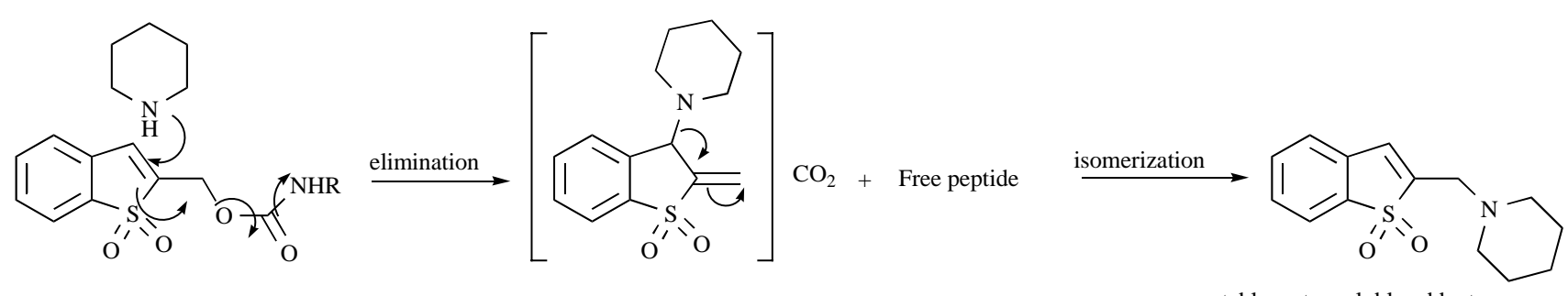

stable water soluble adduct

Scheme 1. Cleavage mechanism of Bsmoc group.

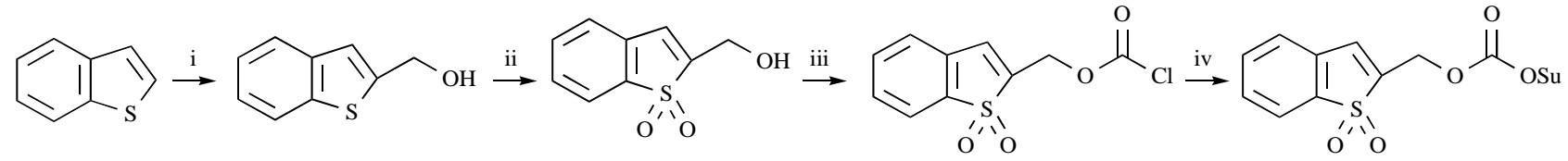

i. n-BuLi, $\left(\mathrm{CH}_{2} \mathrm{O}\right)$ n, $-78^{\circ} \mathrm{C}$; ii. m-CPBA/DCM;

iii. Triphosgene, $0^{\circ} \mathrm{C}$; iv. N-hydroxysuccnimide, DCM

Scheme 2. Synthesis of Bsmoc-Cl. 


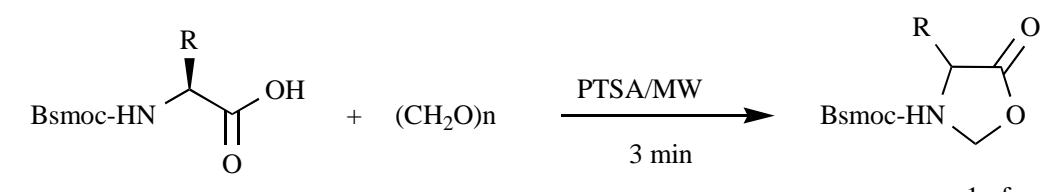

$\mathrm{R}=\mathrm{H}, \mathrm{CH}_{3}, \mathrm{CH}\left(\mathrm{CH}_{3}\right)_{2}, \mathrm{CH}_{2} \mathrm{C}_{6} \mathrm{H}_{5}$,

$\mathrm{CH}_{2} \mathrm{CH}\left(\mathrm{CH}_{3}\right)_{2}, \mathrm{CH}\left(\mathrm{C}_{2} \mathrm{H}_{5}\right) \mathrm{CH}_{3}$

$1 \mathrm{a}-\mathrm{f}$

Scheme 4. Synthesis of Bsmoc -5-oxazolidinones.

Table 1. List of Bsmoc-5-Oxazolidinones

\begin{tabular}{|c|c|c|c|c|c|}
\hline Entry & $\begin{array}{c}\text { Bsmoc-5-oxazolidinones } \\
\text { R }\end{array}$ & $\begin{array}{c}\text { Yield" }^{*} \\
(\%)\end{array}$ & $\begin{array}{c}\mathrm{IR} \\
\left(\mathrm{cm}^{-1}\right)\end{array}$ & $\begin{array}{l}\text { M.P. } \\
\left({ }^{\circ} \mathrm{C}\right)\end{array}$ & $\begin{array}{c}{[\alpha]^{25}} \\
\left(\mathrm{C} 1, \mathrm{CHCl}_{3}\right)\end{array}$ \\
\hline 1a & $\mathrm{H}$ & 91 & 1801,1718 & $100-03$ & - \\
\hline $1 b$ & $\mathrm{CH}_{3}$ & 93 & 1801,1720 & Gum & +79.8 \\
\hline $1 \mathrm{c}$ & $\mathrm{CH}_{2} \mathrm{C}_{6} \mathrm{H}_{5}$ & 92 & 1799,1719 & $95-97$ & +91.9 \\
\hline 1d & $\mathrm{CH}\left(\mathrm{CH}_{3}\right) \mathrm{CH}_{2} \mathrm{CH}_{3}$ & 92 & 1799,1717 & $123-25$ & +74.8 \\
\hline $1 \mathrm{e}$ & $\mathrm{CH}_{2} \mathrm{CH}\left(\mathrm{CH}_{3}\right)_{2}$ & 95 & 1800,1720 & $40-42$ & +81.4 \\
\hline 1f & $\mathrm{CH}\left(\mathrm{CH}_{3}\right)_{2}$ & 95 & 803,1720 & $120-22$ & +77.3 \\
\hline
\end{tabular}

*The reaction time was 3 minutes for all the entries

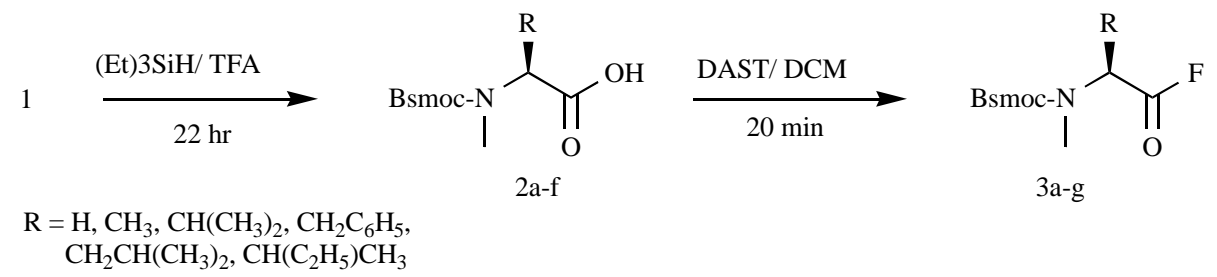

Scheme 5. Reduction of N-Bsmoc-5-oxazolidinones to $N$-methyl amino acids and convertion into corresponding acid fluorides.

Table 2. List of Bsmoc- $N$-Methyl Amino Acids

\begin{tabular}{|c|c|c|c|c|}
\hline Entry & Bsmoc-NMe-amino acids & Yield (\%) & $\begin{array}{l}\text { M.P. } \\
\left({ }^{\circ} \mathrm{C}\right)\end{array}$ & $\begin{array}{c}{[\alpha]^{25}} \\
\left(c \mathrm{~d}, \mathrm{CHCl}_{3}\right)\end{array}$ \\
\hline $2 \mathrm{a}$ & Bsmoc-Sar-OH & 80 & gum & - \\
\hline $2 b$ & Bsmoc-MeAla-OH & 91 & $140-42$ & -27.9 \\
\hline $2 \mathrm{c}$ & Bsmoc-MePhe-OH & 89 & $95-97$ & -20.0 \\
\hline $2 \mathrm{e}$ & Bsmoc-MeLeu-OH & 93 & $40-42$ & -26.8 \\
\hline $2 \mathrm{f}$ & Bsmoc-MeVal-OH & 92 & $105-08$ & -38.9 \\
\hline
\end{tabular}

amino acids showed the presence of singlet peak at around $\delta$ 3.0 ppm pertaining to $\mathrm{N}$-methyl protons.

On treatment of Bsmoc- $N$-methyl amino acids with DAST in DCM resulted in Bsmoc- $N$-methyl amino acid fluorides 3a-g in good yield (Scheme 5, Table 3). The IR analysis of acid fluorides showed a strong stretching absorption band at $1780 \mathrm{~cm}^{-1}$.

Potassium Salt of 7-Aza-1-hydroxybenzotriazole (KOAt), introduced by Sureshbabu and Gopi, was used to mediate the coupling of Bsmoc $-N$-methyl amino acids to prepare dipeptides (Scheme 6, Table 4). The coupling was carried out in $\mathrm{CH}_{2} \mathrm{Cl}_{2}$ at r.t. and was found be free from racemisation. All the isolated peptides were fully charactaerized. In order to test susceptibility of the method towards racemization, the synthesis of two diastereomeric dipeptides Bsmoc- $L$-Phg-Phe-OMe and Bsmoc- $D$-Phg-Phe-OMe was carried out. Bsmoc- $L$ or $D-\mathrm{Phg}-\mathrm{F}$ was coupled with $\mathrm{H}_{2} \mathrm{~N}$ Phe-OMe in presence of KOAt. The ${ }^{1} \mathrm{H}$ NMR analysis of the two diastereomers revealed that the $\mathrm{C}$-methyl doublets of the 
dipeptides differed from each other and also the methyl ester singlet was separated $(\delta 3,59 ; 3,66)$ by $0.07 \mathrm{ppm}$. Further, the HPLC studies of these dipeptides Bsmoc- $L$-Phg-PheOMe $\left(\mathrm{R}_{t}\right.$ value 9.7$)$ and Bsmoc- $D$-Phg-Phe-OMe $\left(\mathrm{R}_{t}\right.$ value 10.7), synthesized by the present method, revealed that they are optically pure and the coupling is completely free from racemization.

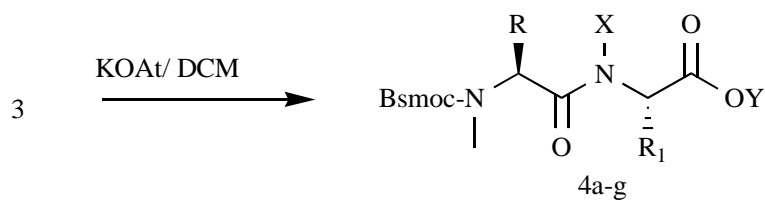

Scheme 6. Synthesis of peptides.

\section{CONCLUSION}

In summary, this paper describes the preparation of Bmoc- $N$-methyl amino acids in two step procedure. Key $N$ Bsmoc-5-oxazolidinones have been isolated in good yields. The reduction of $\mathrm{N}$-Bsmoc-5-oxazolidinones was carried using $\mathrm{Et}_{3} \mathrm{SiH}$ and TFA in $\mathrm{CH}_{2} \mathrm{Cl}_{2}$ at r.t. Bsmoc- $N$-methyl amino acid fluorides are prepared using DAST which are isolated in almost quantitative yield. The coupling of Bsmoc$N$-methyl amino acids was accomplished by KOAt successfully.

\section{EXPERIMENTAL PROCEDURES}

All the reactions were monitored by TLC with precoated silica gel plates purchased from Merk. Column chromatography was performed with Merk silica gel (100-240) at normal atmospheric pressure. The optical rotations were determined with an automatic digital AA-10 polarimeter (Optical activity, U.K). Analytical HPLC was performed on a Shimadzu Class VP- V6.1 with PDA detector; Column: Agilent Zorbax C-18, 250 x 4.0mm column; Mobile phase: $\mathrm{CH}_{3} \mathrm{CN}$ $(65 \%):: \mathrm{H}_{2} \mathrm{O}(35 \%)$; Absorbance : $230 \mathrm{~nm}$; flow rate : 1 $\mathrm{mL} / \mathrm{min} .{ }^{1} \mathrm{H}$ NMR was recorded on a Bruker AMX $400 \mathrm{MHz}$ instrument with $\mathrm{Me}_{4} \mathrm{Si}$ as an internal standed. Mass spectra were recorded on Kratos PCKompact SEQ V1.2.2 spectrometer.

\section{General Procedure for the Synthesis of Bsmoc-Amino Acids}

To a suspension of amino acid $(5 \mathrm{mmol})$ in DCM (10 $\mathrm{mL})$, NMM (0.55 mL, $5 \mathrm{mmol})$ and TMS-Cl $(0.9 \mathrm{~mL}, 12$ $\mathrm{mmol})$ were added in one portion. The reaction mixture was refluxed for $2 \mathrm{hr}$ and cooled in an ice bath. Bsmoc-OSu (1.29 $\mathrm{g}, 5 \mathrm{mmol}$ ) was added at that temperature over a period of 10 min. The reaction mixture was stirred for $1-1.5 \mathrm{hr}$ at r.t. The solvent was removed in vacuo and the resulting oil was distributed between diethyl ether and $10 \% \mathrm{NaHCO}_{3}$ solution. The combined aqueous layers were acidified to $\mathrm{pH} 2$ with

Table 3. List of the Acid Fluorides

\begin{tabular}{|c|c|c|c|c|}
\hline Entry & Bsmoc-amino acids & $\begin{array}{c}\text { Yield } \\
(\mathbf{\%})\end{array}$ & $\begin{array}{c}\text { M.P. } \\
\left({ }^{\mathbf{0}} \mathbf{C}\right)\end{array}$ \\
\hline \hline $3 \mathrm{a}$ & Bsmoc-Nva-F & 89 & $70-72$ & -6.3 \\
\hline $3 \mathrm{~b}$ & Bsmoc-Sar-F & 79 & Gum & - \\
\hline $3 \mathrm{c}$ & Bsmoc-MeAla-F & - & $140-42$ & -6.8 \\
\hline $3 \mathrm{~d}$ & Bsmoc-Me-Leu-F & 91 & Gum & -11.5 \\
\hline 3e & Bsmoc-Me-Ile-F & 82 & Gum & -14.1 \\
\hline $3 \mathrm{f}$ & Bsmoc-Me-Val-F & 90 & $100-02$ & 12.7 \\
\hline $3 \mathrm{~g}$ & Bsmoc-Me-Phe-F & 89 & $110-02$ & -17.8 \\
\hline
\end{tabular}

Table 4. List of the Bsmoc Dipeptides

\begin{tabular}{|c|c|c|c|c|c|c|}
\hline Entry & $\mathbf{R}$ & $\mathbf{R}_{1}$ & $\mathbf{X}$ & $\mathbf{Y}$ & $\begin{array}{c}\text { Yield } \\
(\%)\end{array}$ & $\begin{array}{c}\text { Mass }^{1} \\
{[\mathrm{M}+\mathrm{Na}]^{+}}\end{array}$ \\
\hline $4 \mathrm{a}$ & $\mathrm{CH}_{2} \mathrm{CH}\left(\mathrm{CH}_{3}\right)_{2}$ & $\mathrm{CH}_{3}$ & $\mathrm{H}$ & $\mathrm{CH}_{2} \mathrm{C}_{6} \mathrm{H}_{5}$ & 89 & 551.3 \\
\hline $4 b$ & $\mathrm{CH} 3$ & $\mathrm{CH}_{2} \mathrm{CH}\left(\mathrm{CH}_{3}\right)_{2}$ & $\mathrm{H}$ & $\mathrm{CH}_{3}$ & 92 & 475.2 \\
\hline $4 \mathrm{c}$ & $\left(\mathrm{CH}_{2}\right)_{2} \mathrm{CH}_{3}$ & $\mathrm{H}$ & $\mathrm{CH}_{3}$ & $\mathrm{CH}_{3}$ & 91 & 461.2 \\
\hline $4 d$ & $\mathrm{CH}_{2} \mathrm{CH}\left(\mathrm{CH}_{3}\right)_{2}$ & $\mathrm{CH}_{2} \mathrm{CH}\left(\mathrm{CH}_{3}\right)_{2}$ & $\mathrm{CH}_{3}$ & $\mathrm{CH}_{3}$ & 89 & 531.3 \\
\hline $4 \mathrm{e}$ & $\mathrm{CH}\left(\mathrm{CH}_{3}\right)_{2}$ & $\left(\mathrm{CH}_{2}\right)_{2} \mathrm{CH}_{3}$ & $\mathrm{CH}_{3}$ & $\mathrm{CH}_{3}$ & 87 & 503.1 \\
\hline $4 \mathrm{f}$ & $\mathrm{CH}_{2} \mathrm{CH}\left(\mathrm{CH}_{3}\right)_{2}$ & $\mathrm{CH}\left(\mathrm{CH}_{3}\right)_{2}$ & $\mathrm{CH}_{3}$ & $\mathrm{CH}_{3}$ & 85 & 517.1 \\
\hline $4 \mathrm{~g}$ & $\mathrm{CH}_{2} \mathrm{C}_{6} \mathrm{H}_{5}$ & $\mathrm{CH}\left(\mathrm{CH}_{3}\right) \mathrm{CH}_{2} \mathrm{CH}_{3}$ & $\mathrm{CH}_{3}$ & $\mathrm{CH}_{3}$ & 89 & 565.2 \\
\hline
\end{tabular}

${ }^{1}$ MS [MALDI-TOF] 
conc $\mathrm{HCl}$ and the resulting Bsmoc-amino acids was extracted with ethyl acetate (10 $\mathrm{mL} \mathrm{X} 3$ times). The combined organic layer was washed with water $(20 \mathrm{~mL}$ X 3 times $)$ and dried over anhydrous $\mathrm{Na}_{2} \mathrm{SO}_{4}$. Evaporation of the solvent in vacuo and recrystallization of the resulting residue from DCM: $n$-hexane (1:1) gave Bsmoc-amino acids in good yield.

\section{General Procedure for the Synthesis of Bsmoc-5- Oxazolidinones 1a-f:}

A slurry of Bsmoc-amino acid (10 mmol), paraformaldehyde $(2 \mathrm{~g})$ and $p$-TsOH $(100 \mathrm{mg})$ in toluene $(10 \mathrm{~mL})$ was subjected to microwave irradiation in an unmodified domestic microwave oven operating at $2450 \mathrm{MHz}$ frequency at its full power. After completion of the reaction, the residue was diluted with $\mathrm{CHCl}_{3}(25 \mathrm{~mL})$, washed with $10 \%$ aqueous Na$\mathrm{HCO}_{3}$ (10 mL X 3 times) and water (10 mL X 3 times). The organic layer was dried over anhydrous $\mathrm{Na}_{2} \mathrm{SO}_{4}$ and the solvent was evaporated in vacuo to obtain the 5-oxazolidinones in almost quantitative yield.

Bsmoc-Ala-5-oxazolidinone 1b. ${ }^{1} \mathrm{H}$ NMR $(400 \mathrm{MHz}$, $\left.\mathrm{CDCl}_{3}\right): \delta(\mathrm{ppm})=1.29(\mathrm{~d}, \mathrm{~J}=6.7,3 \mathrm{H}), 3.92(\mathrm{~m}, 1 \mathrm{H}), 4.54$ $(\mathrm{s}, 2 \mathrm{H}), 5.17(\mathrm{~s}, 2 \mathrm{H}), 7.20-7.80(\mathrm{~m}, 5 \mathrm{H}) ;{ }^{13} \mathrm{C}$ NMR $(100$ $\left.\mathrm{MHz}, \mathrm{CDCl}_{3}\right)$ 18.2, 55.3, 65.9, 71.2, 120.8, 122.2, 123.1, 127.5, 130.1, 132.2, 137.5, 139.3, 159.2, 169.1; ES-MS Cald for $\mathrm{C}_{14} \mathrm{H}_{13} \mathrm{NO}_{6} \mathrm{~S} \mathrm{~m} / \mathrm{z}: 324.3[\mathrm{M}+1]^{+}$, found: $324.3[\mathrm{M}+1]^{+}$.

Bsmoc-Phe-5-oxazolidinone 1c. ${ }^{1} \mathrm{H}$ NMR $(400 \mathrm{MHz}$, $\left.\mathrm{CDCl}_{3}\right): \delta(\mathrm{ppm})=2.85-3.05(\mathrm{~m}, 2 \mathrm{H}), 4.50(\mathrm{~s}, 2 \mathrm{H}), 4.65(\mathrm{~m}$, $1 \mathrm{H}), 5.21(\mathrm{~s}, 2 \mathrm{H}), 7.15-7.95(\mathrm{~m}, 10 \mathrm{H}) ;{ }^{13} \mathrm{C} \mathrm{NMR}(100 \mathrm{MHz}$, $\left.\mathrm{CDCl}_{3}\right)$ 31.2, 57.5, 69.0, 72.5, 120.5, 122.4, 123.1, 123.9, 124.6, 125.3, 126.1, 127.1, 127.5, 130.1, 132.2, 137.2, 137.5, 139.3, 162.2, 170.1; ES-MS Cald for $\mathrm{C}_{20} \mathrm{H}_{17} \mathrm{NO}_{6} \mathrm{~S}$ m/z: 400.4 $[\mathrm{M}+1]^{+}$, found: $400.3[\mathrm{M}+1]^{+}$.

Bsmoc-Ile-5-oxazolidinone 1d. ${ }^{1} \mathrm{H}$ NMR $(400 \mathrm{MHz}$, $\left.\mathrm{CDCl}_{3}\right): \delta(\mathrm{ppm})=0.86-1.05(\mathrm{~m}, 6 \mathrm{H}), 1.59-1.63(\mathrm{~m}, 3 \mathrm{H})$, $4.56(\mathrm{~s}, 2 \mathrm{H}), 4.70(\mathrm{~m}, 1 \mathrm{H}), 5.20(\mathrm{~s}, 2 \mathrm{H}), 7.20-7.85(\mathrm{~m}, 5 \mathrm{H})$; ${ }^{13} \mathrm{C}$ NMR $\left(100 \mathrm{MHz}, \mathrm{CDCl}_{3}\right)$ 14.2, 17.5, 27.0, 35.1, 66.5, 69.8, 75.5, 120.7, 122.8, 123.9, 124.6, 126.1, 130.1, 137.2, 162.2, 170.1; ES-MS Cald for $\mathrm{C}_{20} \mathrm{H}_{17} \mathrm{NO}_{6} \mathrm{~S} \mathrm{~m} / \mathrm{z}$ : 400.4 $[\mathrm{M}+1]^{+}$, found: $400.3[\mathrm{M}+1]^{+}$.

Bsmoc-Leu-5-oxazolidinone 1e. ${ }^{1} \mathrm{H}$ NMR $(400 \mathrm{MHz}$, $\left.\mathrm{CDCl}_{3}\right): \delta(\mathrm{ppm})=0.95(\mathrm{~m}, 6 \mathrm{H}), 1.29-1.53(\mathrm{~m}, 3 \mathrm{H}), 4.60(\mathrm{~s}$, $2 \mathrm{H}), 4.75(\mathrm{~m}, 1 \mathrm{H}), 5.25(\mathrm{~s}, 2 \mathrm{H}), 7.18-7.80(\mathrm{~m}, 5 \mathrm{H}) ;{ }^{13} \mathrm{C}$ NMR (100 MHz, $\left.\mathrm{CDCl}_{3}\right)$ 14.2, 17.5, 27.0, 35.1, 66.5, 69.8, 75.5, 120.7, 122.8, 123.9, 124.6, 126.1, 130.1, 137.2, 162.2, 170.1; ES-MS Cald for $\mathrm{C}_{17} \mathrm{H}_{19} \mathrm{NO}_{6} \mathrm{~S} \mathrm{~m} / \mathrm{z}$ : $366.4[\mathrm{M}+1]^{+}$, found: $366.3[\mathrm{M}+1]^{+}$.

Bsmoc-Val-5-oxazolidinone 1f. ${ }^{1} \mathrm{H}$ NMR (400MHz, $\left.\mathrm{CDCl}_{3}\right): \delta(\mathrm{ppm})=1.15(\mathrm{~d}, \mathrm{~J}=6.7,6 \mathrm{H}), 1.71-1.80(\mathrm{~m}, 1 \mathrm{H})$, $4.10(\mathrm{~m}, 1 \mathrm{H}), 4.55(\mathrm{~s}, 2 \mathrm{H}), 5.15(\mathrm{~s}, 2 \mathrm{H}), 7.21-7.80(\mathrm{~m}, 5 \mathrm{H})$; ${ }^{13} \mathrm{C}$ NMR $\left(100 \mathrm{MHz} \mathrm{CDCl}_{3}\right)$ 21.1, 21.3, 27.6, 65.5, 70.1, 79.2, 120.4, 122.9, 124.0, 126.1, 127.2, 129.1, 130.5, 138.2, 162.5, 171.1; ES-MS Cald for $\mathrm{C}_{16} \mathrm{H}_{17} \mathrm{NO}_{6} \mathrm{~S} \mathrm{~m} / \mathrm{z}$ : 352.4 $[\mathrm{M}+1]^{+}$, found: $352.4[\mathrm{M}+1]^{+}$.

General Procedure for the Synthesis of Bsmoc- $N$-Methyl Amino Acids 2a-f:

Bsmoc-5-oxazolidinone $(10 \mathrm{mmol})$ was dissolved in $\mathrm{CHCl}_{3}(15 \mathrm{~mL})$. To this solution was added TFA $(15 \mathrm{~mL})$ and $\mathrm{Et}_{3} \mathrm{SiH}(3 \mathrm{~mL})$ and stirred at r.t. for $24 \mathrm{hr}$. After completion of the reaction mixture, it was concentrated in vacuo and the residue was portioned between ether $(20 \mathrm{~mL})$ and $10 \%$ aqueous $\mathrm{NaHCO}_{3}(20 \mathrm{~mL})$. The combined aqueous layer was acidified with concentrated $\mathrm{HCl}$ and extracted with EtOAc (10 mL X 3 times). The combined organic layer was washed with water $(30 \mathrm{~mL})$ and dried over anhydrous $\mathrm{NaSO}_{4}$. Evaporation of the solvent resulted in Bsmoc- $N$-methyl amino acids in good yield.

Bsmoc-Sar-OH 2a. ${ }^{1} \mathrm{H}$ NMR ( $\left.400 \mathrm{MHz}, \mathrm{CDCl}_{3}\right)$ : $\delta$ $(\mathrm{ppm})=3.02(\mathrm{~s}, 3 \mathrm{H}), 3.60(\mathrm{~s}, 2 \mathrm{H}), 5.25(\mathrm{~s}, 2 \mathrm{H}), 7.25-7.82$ $(\mathrm{m}, 5 \mathrm{H}) ;{ }^{13} \mathrm{C}$ NMR $\left(100 \mathrm{MHz}, \mathrm{CDCl}_{3}\right)$ 32.5, 55.2, 67.5, 119.1, 120.7, 121.5, 126.8, 130.1, 132.2, 137.6, 139.8, 152.2, 172.2; ES-MS Cald for $\mathrm{C}_{13} \mathrm{H}_{13} \mathrm{NO}_{6} \mathrm{~S} \mathrm{~m} / \mathrm{z}$ : $311.1[\mathrm{M}+1]^{+}$, found: $312.2[\mathrm{M}+1]^{+}$.

Bsmoc-MeAla-OH 2b. ${ }^{1} \mathrm{H}$ NMR $\left(400 \mathrm{MHz}, \mathrm{CDCl}_{3}\right): \delta$ $(\mathrm{ppm})=1.29(\mathrm{~d}, \mathrm{~J}=6.7,3 \mathrm{H}), 2.98(\mathrm{~s}, 3 \mathrm{H}), 4.35(\mathrm{~m}, 1 \mathrm{H}), 5.21$ $(\mathrm{s}, 2 \mathrm{H}), 7.15-7.70(\mathrm{~m}, 5 \mathrm{H}) ;{ }^{13} \mathrm{C}$ NMR $\left(100 \mathrm{MHz}, \mathrm{CDCl}_{3}\right)$ 15.1, 35.1, 55.9, 69.2, 119.5, 123.5, 126.1, 126.9, 129.5, 132.5, 138.1, 138.9, 160.5, 172.1; ES-MS Cald for $\mathrm{C}_{14} \mathrm{H}_{15} \mathrm{NO}_{6} \mathrm{~S} \mathrm{~m} / \mathrm{z}: 325.1[\mathrm{M}+1]^{+}$, found: $326.3[\mathrm{M}+1]^{+}$.

Bsmoc-MePhe-OH 2c. ${ }^{1} \mathrm{H}$ NMR (400MHz, $\left.\mathrm{CDCl}_{3}\right): \delta$ $(\mathrm{ppm})=3.00(\mathrm{~s}, 3 \mathrm{H}), 3.21(\mathrm{~m}, 2 \mathrm{H}), 4.25(\mathrm{~m}, 1 \mathrm{H}), 5.30(\mathrm{~s}$, $2 \mathrm{H}), 7.10-7.95(\mathrm{~m}, 10 \mathrm{H}) ;{ }^{13} \mathrm{C}$ NMR $\left(100 \mathrm{MHz} \mathrm{CDCl}_{3}\right) 32.1$, 33.8 , 59.7, 69.0, 119.5, 120.9, 121.5, 121.4, 122.7, 122.9, 123.4, 125.9, 126.1, 129.0, 131.9, 132.0, 132.6, 159.2, 174.1; ES-MS Cald for $\mathrm{C}_{20} \mathrm{H}_{19} \mathrm{NO}_{6} \mathrm{~S} \mathrm{~m} / \mathrm{z}$ : $401.1[\mathrm{M}+1]^{+}$, found: $402.2[\mathrm{M}+1]^{+}$.

Bsmoc-MeIle-OH 2d. ${ }^{1} \mathrm{H}$ NMR $\left(400 \mathrm{MHz}, \mathrm{CDCl}_{3}\right): \delta$ $(\mathrm{ppm})=0.85-1.15(\mathrm{~m}, 8 \mathrm{H}), 2.25(\mathrm{~m}, 1 \mathrm{H}), 2.95(\mathrm{~s}, 3 \mathrm{H}), 4.45$ $(\mathrm{m}, 1 \mathrm{H}), 5.10(\mathrm{~s}, 2 \mathrm{H}), 7.20-7.85(\mathrm{~m}, 5 \mathrm{H}) ;{ }^{13} \mathrm{C}$ NMR $(100$ $\mathrm{MHz}_{\mathrm{CDCl}}$ ) 13.2, 16.6, 26.9, 33.0, 62.5, 67.8, 121.4, 121.9, 125.4, 127.2, 128.0, 129.5, 131.1, 138.1, 159.0, 173.1; ESMS Cald for $\mathrm{C}_{17} \mathrm{H}_{21} \mathrm{NO}_{6} \mathrm{~S} \mathrm{~m} / \mathrm{z}$ : $367.1[\mathrm{M}+1]^{+}$, found: 368.0 $[\mathrm{M}+1]^{+}$.

Bsmoc-MeLeu-OH 2e. ${ }^{1} \mathrm{H}$ NMR (400MHz, $\left.\mathrm{CDCl}_{3}\right): \delta$ $(\mathrm{ppm})=0.92(\mathrm{~d}, \mathrm{~J}=6.7,6 \mathrm{H}), 1.29(\mathrm{~m}, 2 \mathrm{H}), 2.10(\mathrm{~m}, 1 \mathrm{H})$, $3.00(\mathrm{~s}, 3 \mathrm{H}), 4.20(\mathrm{~m}, 1 \mathrm{H}), 5.17(\mathrm{~s}, 2 \mathrm{H}), 7.18-7.80(\mathrm{~m}, 5 \mathrm{H})$; ${ }^{13} \mathrm{C}$ NMR (100 MHz, $\left.\mathrm{CDCl}_{3}\right)$ 18.2, 22.0, 27.1, 33.7, 65.5, $67.8,121.0,122.7,123.1,123.9,127.7,133.2,135.6,137.2$, 165.2, 172.4; ES-MS Cald for $\mathrm{C}_{17} \mathrm{H}_{21} \mathrm{NO}_{6} \mathrm{~S} \mathrm{~m} / \mathrm{z}$ : 367.1 $[\mathrm{M}+1]^{+}$, found: $368.4[\mathrm{M}+1]^{+}$.

Bsmoc-MeVal-OH 2f. ${ }^{1} \mathrm{H}$ NMR $\left(400 \mathrm{MHz}, \mathrm{CDCl}_{3}\right): \delta$ $(\mathrm{ppm})=0.95(\mathrm{~d}, \mathrm{~J}=6.7,6 \mathrm{H}), 2.22(\mathrm{~m}, 1 \mathrm{H}), 2.98(\mathrm{~s}, 3 \mathrm{H}), 4.35$ $(\mathrm{m}, 1 \mathrm{H}), 5.15(\mathrm{~s}, 2 \mathrm{H}), 7.21-7.75(\mathrm{~m}, 5 \mathrm{H}) ;{ }^{13} \mathrm{C}$ NMR $(100$ $\left.\mathrm{MHz}, \mathrm{CDCl}_{3}\right)$ 15.3, 29.2, 33.3, 67.3, 72.1, 121.1, 122.0, $123.4,125.9,129.0,132.1,132.9,133.4,165.5,173.7$; ESMS Cald for $\mathrm{C}_{16} \mathrm{H}_{19} \mathrm{NO}_{6} \mathrm{~S} \mathrm{~m} / \mathrm{z}$ : $353.1[\mathrm{M}+1]^{+}$, found: 354.3 $[\mathrm{M}+1]^{+}$.

\section{Bsmoc- $N$-Methyl Amino Acid Fluorides 3a-g:}

To a suspension of Bsmoc- $N$-methyl amino acid (1 mmol) in DCM (5 mL) was added DAST $(0.2 \mathrm{~mL}, 1.5$ mmole) under nitrogen and stirred for an hr at r.t. After the completion of the reaction, it was further diluted with DCM $(10 \mathrm{~mL})$ and washed with cold water $(10 \mathrm{~mL} \mathrm{X} 2$ times). The solvent was dried over anhydrous $\mathrm{Na}_{2} \mathrm{SO}_{4}$ and was evaporated under reduced pressure to result in a residue which was 
recrystallized using DCM: n-hexane to yield Bsmoc-amino acid fluorides as solids.

\section{General Procedure for the Coupling of Bsmoc- $N$-Methyl Amino Acid Fluorides Mediated by KOAt 4a-g}

To a stirred solution of Bsmoc- $N$-methyl amino acid fluoride (2 mmole) and KOAt (1.1 mmole) in dry DCM (5 $\mathrm{mL}$ ) was added a solution of amino free amino acid ester in dry DCM $(5 \mathrm{~mL})$ and the reaction mixture was stirred at r.t. The progress of the reaction was monitored by TLC. After the completion of the reaction, it was concentrated and the resulting residue was diluted with $\mathrm{CH}_{2} \mathrm{Cl}_{2}(10 \mathrm{~mL})$, washed thrice with $5 \mathrm{~mL}$ portions of $5 \% \mathrm{HCl}, 5 \% \mathrm{NaHCO}_{3}$ and water and dried over anhydrous $\mathrm{Na}_{2} \mathrm{SO}_{4}$. Evaporation of the solvent in vасио and recrystallization of the residue from $\mathrm{CH}_{2} \mathrm{Cl}_{2}$ : petroleum ether mixture $(1: 3)$ yielded the peptide.

Bsmoc-Nmethyl-Leu-Ala-OBzl 4a. ${ }^{1} \mathrm{H}$ NMR $(400 \mathrm{MHz}$, $\left.\mathrm{CDCl}_{3}\right): \delta(\mathrm{ppm})=0.82-1.08(\mathrm{~m}, 6 \mathrm{H}), 1.31(\mathrm{~d}, \mathrm{~J}=5.9 \mathrm{~Hz}$, $3 \mathrm{H}), 1.51(\mathrm{~m}, 2 \mathrm{H}), 1.72(\mathrm{~m}, 1 \mathrm{H}), 2.98(\mathrm{~s}, 3 \mathrm{H}), 4.49(\mathrm{~m}, 1 \mathrm{H})$, $4.68(\mathrm{~m}, 1 \mathrm{H}), 4.97-5.28(\mathrm{~m}, 3 \mathrm{H}), 5.35(\mathrm{~s}, 2 \mathrm{H}), 7.32-7.77(\mathrm{~m}$, $10 \mathrm{H}$ ); MS (MALDI-TOF) calcd. for $\mathrm{C}_{27} \mathrm{H}_{32} \mathrm{~N}_{2} \mathrm{O}_{7} \mathrm{~S} \mathrm{~m} / \mathrm{z}$ : 551.2 $[\mathrm{M}+\mathrm{Na}]^{+}, 567.7[\mathrm{M}+\mathrm{K}]^{+}$, found: $551.3[\mathrm{M}+\mathrm{Na}]^{+}, 567.5$ $[\mathrm{M}+\mathrm{K}]^{+}$; Anal. Calcd for $\mathrm{C}_{27} \mathrm{H}_{32} \mathrm{~N}_{2} \mathrm{O}_{7} \mathrm{~S}(\%)$ : C, 61.34; $\mathrm{H}$, 6.10 ; N, 5.29; found: C, 61.21; H, 6.01; N, 5.14.

Bsmoc-NMeVal-Leu-OMe 4b. ${ }^{1} \mathrm{H}$ NMR (400 MHz, $\left.\mathrm{CDCl}_{3}\right): \delta(\mathrm{ppm})=0.89-1.05(\mathrm{~m}, 6 \mathrm{H}), 1.35(\mathrm{~d}, \mathrm{~J}=5.7,3 \mathrm{H})$, 1.55-1.75 (m, 3H), $3.00(\mathrm{~s}, 3 \mathrm{H}), 3.60(\mathrm{~s}, 3 \mathrm{H}), 4.54(\mathrm{~m}, 1 \mathrm{H})$ 5.13-5.29 (m, 3H), $6.32(\mathrm{br}, 1 \mathrm{H}), 7.35-7.80(\mathrm{~m}, 4 \mathrm{H}) \mathrm{MS}$ (MALDI-TOF) calcd. for $\mathrm{C}_{21} \mathrm{H}_{28} \mathrm{~N}_{2} \mathrm{O}_{7} \mathrm{~S} \mathrm{~m} / \mathrm{z}: 475.2[\mathrm{M}+$ $\mathrm{Na}]^{+}, 491.1[\mathrm{M}+\mathrm{K}]^{+}$, found: $475.2[\mathrm{M}+\mathrm{Na}]^{+}, 491.1[\mathrm{M}+$ $\mathrm{K}]^{+}$.

Bsmoc-Nva-Sar-OMe 4c. ${ }^{1} \mathrm{H}$ NMR $\left(400 \mathrm{MHz}, \mathrm{CDCl}_{3}\right)$ : $\delta(\mathrm{ppm})=0.85(\mathrm{t}, \mathrm{J}=6.7,3 \mathrm{H}), 1.32-167(\mathrm{~m}, 4 \mathrm{H}), 2.97(\mathrm{~s}$, $3 \mathrm{H}), 3.01(\mathrm{~s}, 3 \mathrm{H}), 3.65(\mathrm{~s}, 3 \mathrm{H}) 4.24(\mathrm{~m}, 1 \mathrm{H}), 5.17(\mathrm{~s}, 2 \mathrm{H})$, $5.65(\mathrm{~m}, 1 \mathrm{H}), 7.30-7.79(\mathrm{~m}, 4 \mathrm{H})$; MS (MALDI-TOF) calcd. for $\mathrm{C}_{20} \mathrm{H}_{26} \mathrm{~N}_{2} \mathrm{O}_{7} \mathrm{~S} \mathrm{~m} / \mathrm{z}: 461.1\left[\mathrm{M}+\mathrm{Na}^{+}, 477.1[\mathrm{M}+\mathrm{K}]^{+}\right.$, found: $461.2[\mathrm{M}+\mathrm{Na}]^{+}, 477.1[\mathrm{M}+\mathrm{K}]^{+}$.

Bsmoc-NMeLeu-NMeLeu-OMe 4d. ${ }^{1} \mathrm{H}$ NMR (400 $\left.\mathrm{MHz}_{\mathrm{CDCl}}\right): \delta(\mathrm{ppm})=0.85-1.01(\mathrm{~m}, 12 \mathrm{H}), 1.27-1.39(\mathrm{~m}$, $3 \mathrm{H}), 1.52-1.76(\mathrm{~m}, 3 \mathrm{H}), 2.95(\mathrm{~s}, 3 \mathrm{H}), 3.01(\mathrm{~s}, 3 \mathrm{H}), 3.63(\mathrm{~s}$, $3 \mathrm{H}), 4.92(\mathrm{~m}, 1 \mathrm{H}) 5.12(\mathrm{~m}, 1 \mathrm{H}), 5.20(\mathrm{~s}, 2 \mathrm{H}), 7.35-7.77(\mathrm{~m}$, $4 \mathrm{H}$ ); MS (MALDI-TOF) calcd. for $\mathrm{C}_{25} \mathrm{H}_{36} \mathrm{~N}_{2} \mathrm{O}_{7} \mathrm{~S} \mathrm{~m} / \mathrm{z}: 531.2$ $[\mathrm{M}+\mathrm{Na}]^{+}, 547.2[\mathrm{M}+\mathrm{K}]^{+}$, found: $531.3[\mathrm{M}+\mathrm{Na}]^{+}, 547.0$ $[\mathrm{M}+\mathrm{K}]^{+}$.

Bsmoc-NMeVal-Nva-OMe 4e. ${ }^{1} \mathrm{H}$ NMR (400 MHz, $\left.\mathrm{CDCl}_{3}\right): \delta(\mathrm{ppm})=0.89(\mathrm{t}, \mathrm{J}=5.9,3 \mathrm{H}), 1.15-1.45(\mathrm{~m}, 10 \mathrm{H})$, $1.97(\mathrm{~m}, 1 \mathrm{H}), 2.97(\mathrm{~s}, 3 \mathrm{H}), 3.05(\mathrm{~s}, 3 \mathrm{H}), 3.67(\mathrm{~s}, 3 \mathrm{H}), 4.74$ $(\mathrm{m}, 1 \mathrm{H}) 5.35(\mathrm{~m}, 1 \mathrm{H}), 5.18(\mathrm{~s}, 2 \mathrm{H}), 7.30-7.75(\mathrm{~m}, 4 \mathrm{H})$; MS (MALDI-TOF) calcd. for $\mathrm{C}_{23} \mathrm{H}_{32} \mathrm{~N}_{2} \mathrm{O}_{7} \mathrm{~S} \mathrm{~m} / \mathrm{z}$ : $503.2[\mathrm{M}+$ $\mathrm{Na}]^{+}, 519.2[\mathrm{M}+\mathrm{K}]^{+}$, found: $503.1[\mathrm{M}+\mathrm{Na}]^{+}, 519.1[\mathrm{M}+$ $\mathrm{K}]^{+}$.

Bsmoc-NMeLeu-NMeVal-OMe 4f. ${ }^{1} \mathrm{H}$ NMR (400 $\left.\mathrm{MHz}, \mathrm{CDCl}_{3}\right): \delta(\mathrm{ppm})=0.85-1.02(\mathrm{~m}, 6 \mathrm{H}), 1.32(\mathrm{~m}, 2 \mathrm{H})$, 1.47-1.68 (m, 7H), $1.85(\mathrm{~m}, 1 \mathrm{H}), 2.95(\mathrm{~s}, 3 \mathrm{H}), 3.02(\mathrm{~s}, 3 \mathrm{H})$, $3.60(\mathrm{~s}, 3 \mathrm{H}), 4.52(\mathrm{~m}, 1 \mathrm{H}) 5.65(\mathrm{~m}, 1 \mathrm{H}), 5.17$ (s, 2H), 7.297.77 (m, 4H); MS (MALDI-TOF) calcd. for $\mathrm{C}_{24} \mathrm{H}_{34} \mathrm{~N}_{2} \mathrm{O}_{7} \mathrm{~S}$ $\mathrm{m} / \mathrm{z}: 517.2[\mathrm{M}+\mathrm{Na}]^{+}, 533.2[\mathrm{M}+\mathrm{K}]^{+}$, found: $517.1[\mathrm{M}+$ $\mathrm{Na}]^{+}, 533.3[\mathrm{M}+\mathrm{K}]^{+}$
Bsmoc-NMePhe-NMeIle-OMe 4g. ${ }^{1} \mathrm{H}$ NMR (400 MHz, $\left.\mathrm{CDCl}_{3}\right): \delta(\mathrm{ppm})=1.01-1.27(\mathrm{~m}, 8 \mathrm{H}), 1.56(\mathrm{~m}, 1 \mathrm{H}), 2.72(\mathrm{~m}$, $2 \mathrm{H}), 2.97(\mathrm{~s}, 3 \mathrm{H}), 3.00(\mathrm{~s}, 3 \mathrm{H}), 3.67(\mathrm{~s}, 3 \mathrm{H}), 4.67(\mathrm{~m}, 1 \mathrm{H})$ $5.32(\mathrm{~m}, 1 \mathrm{H}), 5.20(\mathrm{~s}, 2 \mathrm{H}), 7.19-7.89(\mathrm{~m}, 10 \mathrm{H}) ; \mathrm{MS}$ (MALDI-TOF) calcd. for $\mathrm{C}_{28} \mathrm{H}_{34} \mathrm{~N}_{2} \mathrm{O}_{7} \mathrm{~S} \mathrm{~m} / \mathrm{z}$ : $565.2[\mathrm{M}+$ $\mathrm{Na}]^{+}, 581.2[\mathrm{M}+\mathrm{K}]^{+}$, found: $565.2[\mathrm{M}+\mathrm{Na}]^{+}, 581.1[\mathrm{M}+$ $\mathrm{K}]^{+}$.

\section{Racemization Studies were Carried Out by Preparing Following Compounds}

\section{Bsmoc-L-Phg-Phe-OMe}

To a stirred solution of Bsmoc-Phg-F ( $0.75 \mathrm{~g}, 2$ mmoles) and KOAt (0.38 g, 2.2 mmoles) in dry DCM (5 mL) was added a solution of $\mathrm{H}_{2} \mathrm{~N}$-Phe-OMe (0.36 g, 2 mmoles) dissolved in dry DCM $(5 \mathrm{~mL})$. The reaction mixture was stirred for $30 \mathrm{~min}$ at r.t. and its workup, following the general procedure yielded the dipeptide as a white crystalline solid (0.93 g, $88 \%)$; m. p. $125-27^{\circ} \mathrm{C}$; TLC $\mathrm{R}_{\mathrm{f}}($ EtOAc : $n$-hexane :: $3: 7)$, $0.43 ;[\alpha]^{25}{ }_{\mathrm{D}}-14.0^{\circ}$ (c $\left.0.5, \mathrm{DMF}\right) ;{ }^{1} \mathrm{H}$ NMR $\left(\mathrm{CDCl}_{3}\right): \delta 2.9$ $(2 \mathrm{H}, \mathrm{d}), 3.64(3 \mathrm{H}, \mathrm{s}), 4.5(1 \mathrm{H}, \mathrm{m}), 5.10(2 \mathrm{H}, \mathrm{d}), 5.25(1 \mathrm{H}, \mathrm{d})$, $6.25(2 \mathrm{H}, 2 \mathrm{~d}), 7.1-7.8$ (15 ArH , m). Anal. Calcd. for $\mathrm{C}_{28} \mathrm{H}_{26} \mathrm{~N}_{2} \mathrm{O}_{7} \mathrm{~S}_{1}$ (534.58): C, 62.91; H, 4.90; N, 5.24; found: C, 62.82; H, 4.81; N, 5.14. MS (MALDI-TOF): $\mathrm{m} / \mathrm{z}$ calcd. $557.5[\mathrm{M}+\mathrm{Na}]^{+}, 573.6[\mathrm{M}+\mathrm{K}]^{+}$, found: $557.7[\mathrm{M}+\mathrm{Na}]^{+}$, $573.1[\mathrm{M}+\mathrm{K}]^{+}$.

\section{Bsmoc-D-Phg-Phe-OMe}

To a stirred solution of Bsmoc-D-Phg-F (0.75 g, 2 mmoles) and KOAt (0.38 g, 2.2 mmoles) in dry DCM (5 $\mathrm{mL})$ was added a solution of $\mathrm{H}_{2} \mathrm{~N}$-Phe-OMe $(0.36 \mathrm{~g}, 2$ mmoles) dissolved in dry DCM $(5 \mathrm{~mL})$. The reaction mixture was stirred for $30 \mathrm{~min}$ at r.t. and its workup yielded the dipeptide as a white crystalline solid $(0.95 \mathrm{~g}, 90 \%), \mathrm{m} . \mathrm{p}$. $127-30^{\circ} \mathrm{C} ;$ TLC R $\mathrm{R}_{f}$ (EtOAc : $n$-hexane :: $\left.3: 7\right), 0.45 ;[\alpha]^{25}$ $+14.4^{\mathrm{o}}$ (c 0.5, DMF); ${ }^{1} \mathrm{H}$ NMR $\left(\mathrm{CDCl}_{3}\right): \delta 3.0(2 \mathrm{H}, \mathrm{d}), 3.73$ $(3 \mathrm{H}, \mathrm{s}), 4.5(1 \mathrm{H}, \mathrm{m}), 5.10(2 \mathrm{H}, \mathrm{d}), 5.25(1 \mathrm{H}, \mathrm{d}), 6.25(2 \mathrm{H}$, two d), 7.1-7.8 (15 ArH , m). Anal. Calcd. for $\mathrm{C}_{28} \mathrm{H}_{26} \mathrm{~N}_{2} \mathrm{O}_{7} \mathrm{~S}_{1}$ (534.58): C, 62.91; H, 4.90; N, 5.24. found: C, 62.85; H, 4.88; N, 5.16. MS (MALDI-TOF): $\mathrm{m} / \mathrm{z}$ calcd. $557.5[\mathrm{M}+$ $\mathrm{Na}]^{+}, 573.6[\mathrm{M}+\mathrm{K}]^{+}$, found: $557.9[\mathrm{M}+\mathrm{Na}]^{+}, 573.5[\mathrm{M}+$ $\mathrm{K}]^{+}$.

\section{ACKNOWLEDGEMENTS}

The authors thank the CSIR, Govt. of India, New Delhi for financial support.

\section{REFERENCES}

[1] Fairlie, D. P., Abbenante, G. and March, D. R. Macrocyclic peptidomimitics forcing peptides into bioactive conformations. (1995) Curr. Med. Chem., 2, 654.

[2] Cody, W. L., He, J. X., Reily, M. D., Haleen, S. J., Walker, D. M., Reyner, E. L., Stewart, B. H. and Doherty, A. M. Design of a potent combined pseudopeptide endothelin-a/endothelin-b receptor antagonist, Ac-DBhg ${ }^{16}$-Leu-Asp-Ile-[NMe] Ile- $\operatorname{Trp}^{21}$ (PD 1562 52): examination of its pharmacokinetic and spectral properties. (1997) J. Med. Chem., 40, 2228.

[3] Haviv, F., Fitzpatrick, T. D., Swenson, R. E., Nichols, C. J., Mort N. A., Bush, E. N., Dim, G., Bammert, G., Nguyen, A., Rhutasel, N. S., Nellans, H. N., Hoffman, D. J., Johnson, E. S. and Greer, J. Effect of $N$-methyl substitution of the peptide bonds in luteinizing hormone-releasing hormone agonists. (1993) J. Med. Chem., 36, 363. 
[4] Ruegger, A., Kuhn, M., Lichti, H., Loosli, H-R., Huguenin, R., Quiquerez, C. and von Wartburg, A. Cyclosporin A, ein immunsuppressiv wirksamer peptidmetabolit a aus trichoderma polysporum (LINK ex PERS.). (1976) Helv. Chim. Acta., 59, 1075.

[5] Gordon, D. J., Sciarretta, K. L. and Meredith, S. C. Inhibition of $\beta$ amyloid (40)fibrillogenesis and disassembly of $\beta$-amyloid (40) fibrils by short $\beta$-amyloid congeners containing $N$-methyl amino acids at alternate residues. (2001) Biochemistry, 40, 8237.

[6] Kapurniotu, A., Schmauder, A. and Tenidis, K. Structure-based design and study of non-amyloidogenic, double N-methylated IAPP amyloid core sequences as inhibitors of IAPP amyloid formation and cytotoxicity. (2002) J. Mol. Biol., 315, 339.

[7] Miller, S. M., Simon, R. J., Ng, S., Zuckermann, R. N., Kerr, J. M. and Moos, W. H. Comparison of the proteolytic susceptibilities of homologous L-amino acid, D-amino acid and N-substituted glycine peptide and peptoid oligomers. (1995) Drug. Dev. Res., 35, 20.

[8] Aurelio, L., Brownlee, R. T. C. and Hughes, A. B. Synthetic preparation of $N$-methyl- $\alpha$-amino acids. (2004) Chem. Rev.,104, 5823.

[9] McDermott, J. R. and Benoiton, N. L. $N$-methylamino acids in peptide synthesis. a new synthesis of $N$-benzyloxycarbonyl, $\mathrm{N}$ methylamino acids. (1973) Can. J. Chem., 51, 1915.

[10] Ben-Ishai, D. Reaction of acylamino acids with paraformaldehyde. (1957) J. Am. Chem. Soc., 79, 5736.

[11] Tantry, S. J., Kantharaju and Sureshbabu, V. V. Microwave accelerated efficient synthesis of $\mathrm{N}$-fluorenylmethoxycarbonyl/tbutoxycarbonyl/benzyloxycarbonyl-5- oxazolidinones (2002) Tetrahedron Lett., 43, 9461.

[12] Govender, T. and Arvidsson, P. I. Facile synthesis of Fmoc-Nmethylated $\alpha$ - and $\beta$ - amino acids. (2006) Tetrahedron Lett., 47, 1691.

[13] Auerbach, J., Zamore, M. F. and Weinreb, S. M. N-methylation of amides, lactams, and ureas. (1976) J. Org. Chem., 41, 725.

[14] Freidinger, R. M., Hinkle, J. S., Perlow, D. S.Synthesis of 9- Fluorenylmethyloxycarbonyl -protected $\mathrm{N}$-alkyl amino acids by reduction of Oxazolidinones. (1983) J. Org. Chem., 48, 77.

[15] Aurelio, L., Brownlee, R. T. C. and Hughes, A. B. A novel synthesis of $N$-methyl Asparagine, Arginine, Histidine and Tryptophan. (2002) Org. Lett., 4, 3767.

[16] Aurelio, L., Box, J. S., Brownlee, R. T. C., Hughes, A. B. and Sleebs, M. M. An efficient synthesis of N-methyl amino acids by way of intermediate 5- oxazolidinones. (2003) J. Org. Chem., 68, 2652.
Zhang, S., Govender, T., Norstrom, T. and Arvidsson, P. I. An improved synthesis of Fmoc- $N$-methyl- $\alpha$-amino acids. (2005) $J$. Org. Chem., 70, 6918.

[18] Carpino, L. A., Ismail, M., Truran, G. A., Mansour, E. M. E., Iguchi, S., Ionescu, D., El-Faham, A., Riemer, C. and Warrass, R. The 1,1-Dioxobenzo[b]thiophene-2-ylmethyloxycarbonyl (Bsmoc) amino- protecting group. (1999) J. Org. Chem., 64, 4324.

[19] Carpino, L. A., Philbin, M., Ismail, M., Mansour, E. M. E., Iguchi, S., Ionescu, D., El-Faham, A., Riemer, C., Warrass, R. and Weiss, M. S. New family of base- and nucleophile-sensitive amino-protecting groups. A michael-acceptor-based deblocking process. practical utilization of the 1,1-Dioxobenzo[b]thiophene-2-ylmethyl oxycarbonyl (Bsmoc) Group. (1997) J. Am. Chem. Soc., 119, 9915.

[20] Carpino, L. A., Ghassemi, S., Ionescu, D., Ismail, M., Sadat, Aalaee, D., Truran, G. A., Mansour, E. M. E., Siwruk, G. A., Eynon, J. S. and Morgan, B. Rapid,continuous solution-phase peptide synthesis :application to peptides of pharmaceutical interest. (2003) Org. Process Res. Dev., 7, 28.

[21] Carpino, L. A. and Mansour, E. S. M. E. Protected beta and gamma aspartic and Glutamicacidfluorides. (1992) J. Org. Chem., 57, 6371.

[22] Mayer, S. C. and Joullie, M. M. Protected beta and gamma aspartic and glutamic Acidfluorides. (1994) Synth. Commun., 24, 2367.

[23] Wenschuh, H., Beyermann, M. and Krause, E. Fmoc amino acid fluorides: convenient reagents for the solid-phase assembly of peptides incorporating sterically hindered residues. (1994) J. Org. Chem., 59, 3275 .

[24] Sureshbabu, V. V. and Ananda, K. Synthesis of peptides employing Fmoc-/Boc-/Z- amino acid fluorides and activated comercial Zinc dust. (2000) Lett. Pept. Sci., 7, 41.

[25] Yokum, T. S., Elzer, P. H., McLaughlin, M. L. Antimicrobial, $\alpha, \alpha-$ Dialkylated amino acid Rich peptides with in-vivo Activity against an Intracellular Pathogen. (1996) J. Med. Chem., 39, 3603.

[26] Wenschuh, H., Beyermann, M., Krause, E., Carpino, L. A. and Bienert, M. Efficient solid phase assembly of peptides bearing contiguous highly hindered Aib residues via Fmoc Aib fluoride. (1993) Tetrahedron Lett., 34, 3733.

[27] Olah, G. A., Nojima, M. and Kerekes, I. Synthetic methods and reactions; IV. fluorination of carboxylic acids with cyanuric fluoride. (1973) Synthesis, 487.

[28] Kaduk, C., Wenschuh, H., Beyermann, M. and Former, K. Synthesis of Fmoc-amino acid fluorides via DAST, an alternative fluorinating agent (1995) Lett. Pept. Sci., 2, 285.
Received: May 07, 2008 Revised: July 29, 2008 Accepted: August 01, 2008 